\title{
The mean-integral representation of rectangles
}

\author{
NEIL A. MACMILLAN and ADAM S. ORNSTEIN \\ Brooklyn College, City University of New York, Brooklyn, New York
}

\begin{abstract}
To assess perceptual interaction between the height and width of rectangles, we used an accuracy variant of the Garner paradigm. We measured the discriminability of height and width (baseline tasks) and size and shape (correlated tasks). From the $d^{\prime}$ values in these conditions, we estimated perceptual distances and inferred a mean-integral representation in which height and width corresponded to nonindependent dimensions in a perceptual space. This model accounted well for performance in these two-stimulus conditions, and it also explained $70 \%-80 \%$ of the decline in performance in selective and divided attention. In a second experiment, conducted for purposes of comparison with the rectangle discrimination Experiment, we studied the discrimination of horizontal and vertical line segments connected in an L-shape. In size discrimination, observers were equally good with line pairs and rectangles, suggesting holistic perception; but in shape discrimination, they appeared to combine information from the two line-pair components of the rectangle independently. The mean-integral model was again successful in relating performance in the Garner tasks quantitatively.
\end{abstract}

Rectangles are among the simplest visual forms, and the manner in which they are perceived is of some importance in visual perception. In addition, many studies of rectangles have been motivated by psychophysical considerations. Complex perceptual objects (faces, say, or speech) are often said to be "multidimensional," meaning that such objects depend perceptually on several characteristics, and rectangle perception has provided a workshop for trying out alternative realizations of the multidimensional idea.

As illustrated by the minimal set in Figure 1, rectangles can differ in height (vertical axis), width (horizontal axis), size (along the minor diagonal), and shape (along the major diagonal). Any or all of these dimensions may be psychologically important, and a critical research question has been whether height and width, or size and shape, are the defining dimensions for perception. A second question has been whether the dimensions used in the representation interact, or whether they are independent. As Schönemann (1990) has pointed out, there has not been full agreement; the answers have depended in part on the psychophysical methodology used to ask the questions.

One useful experimental approach has been to measure identification (absolute judgment) of sets of rectangles. Weintraub (1971) combined 15 values of height and 15 values of width to form a group of 225 forms. Subsets of 15 , one for each possible height, were constructed in a variety of ways, and stimuli within each subset were pre-

This research was supported by NSF grant DBS 92-12043 and a PSC-CUNY award to the first author. A preliminary report of the data was presented at the Psychonomic Society meetings in Washington, DC, November 1993. We are grateful to John Kingston and Todd Maddox for their comments on an earlier draft. Please address correspondence to N. A. Macmillan, Department of Psychology, Brooklyn College, Brooklyn, NY 11210 (e-mail: nmacmill@broadway.gc.cuny.edu). sented in random order for identification. Subjects were asked to judge height, although this was always redundant with width in some way. Transmitted information was 2.65 bits when the width and height were negatively correlated (so that the rectangles differed in shape) but only 2.0 bits when they were positively correlated (so that the rectangles were squares differing in size). Best performance ( 2.7 bits) was found with a subset in which values of width and height were combined randomly. Weintraub interpreted the differences between conditions as arising from differences in the extent to which relativistic information about width was useful in making the height judgments.

Monahan and Lockhead (1977) noted that the perceptual distance between stimuli was largest in Weintraub's random condition and hypothesized (following Lockhead, 1970) that perceptual distance was the key to predicting identification performance for redundant sets like those used by Weintraub. To test this proposal, Monahan and Lockhead compared identification for two subsets of rectangles, one differing only in size, the other chosen to maximize distance. The maximal-distance set produced better performance, in terms of both percent correct and response time. In a second experiment, the stimuli were pairs of vertical lines. Perceptual distance was directly estimated by applying multidimensional scaling (MDS) to dissimilarity judgments and it was found to correlate very highly with confusion errors in identification. Monahan and Lockhead concluded that "it is the separation between stimuli in similarity space and not the experimenterdefined differences between stimuli that predicts objectidentification performance" (p. 94).

The MDS technique has also been used by itself to construct a perceptual representation. Krantz and Tversky (1975) inferred a representation in which the relevant perceptual dimensions were approximately size and shape but found an interaction (an "augmentation effect") in which 


\section{One set of rectangle stimuli}

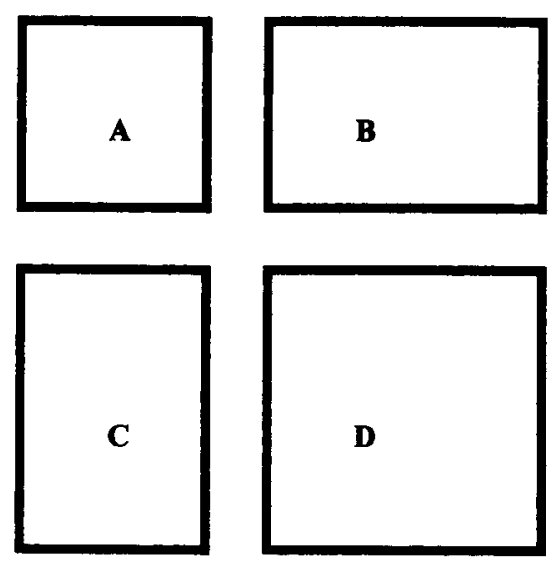

Figure 1. Four rectangles that differ in height and width (and also in size and shape). One set of stimuli in Experiment 1 was similar to these, except that the differences along all dimensions were small, about a JND.

shape differences appeared greater for larger rectangles. Borg and Leutner (1983) confirmed this interaction with a set of rectangles formed by orthogonally varying area and shape but found independence for a set varying in height and width, suggesting that these were the perceptually relevant dimensions. Wiener-Ehrlich (1978) found interactions for both a height/ width set, in which apparent width increased with height, and a size/shape set, in which the interaction had no simple description.

One possible reason for the disagreement among these very similar studies has been noted by Schönemann, Dorcey, and Kienapple (1985). To infer a spatial representation with the use of MDS, it is necessary to know the "metric" by which the distance between points is measured. In MDS, an arbitrary monotonic function is used to transform the dissimilarity measure to a distance, and the metric may be any member of the Minkowski class, of which the city-block and Euclidean metrics are the best-known examples. Schönemann et al. demonstrated, however, that a change in the monotonic transformation can change the appropriate metric. Of the MDS papers described earlier, Borg and Leutner concluded that a city-block metric was best, whereas WienerEhrlich (1978) favored the Euclidean rule.

Two solutions to this difficulty have been offered. Schönemann and his colleagues (Lazarte \& Schönemann, 1991; Schönemann et al., 1985; Schönemann \& Lazarte, 1987) have developed a method for inferring the metric from the actual dissimilarity judgments (which are not subject to arbitrary transformation). The metrics that they infer are not members of the Minkowski class and are not the same for all observers. A second tack, often taken in conjunction with MDS, has been the application of measurement theory (Dunn, 1983; Krantz \& Tversky, 1975; Wender, 1971) to test the axioms of the metric-space representation. Frequently these axioms are found to fail - the augmentation effect in Krantz and Tversky (1975) is an example-but the implications of this failure for the "perceptual representation" are not clear.

\section{The Garner Paradigm}

A method that avoids some of the complexities of dissimilarity judgments is the Garner (1974) paradigm, in which several classification tasks are applied to the same $2 \times 2$ stimulus set. In this paradigm, a stimulus array is constructed by varying two stimulus dimensions orthogonally, as in Figure 1, and observers are required to discriminate various subsets from the array. If we denote the stimuli by the letters $A, B, C$, and $D$, as in Figure 1, we can summarize the four distinct tasks of the Garner paradigm as follows:

1. In baseline conditions, the two possible stimuli differ either just in height or just in width. There are four such tasks: $A$ versus $C$ and $B$ versus $D$ for height discrimination, and $A$ versus $B$ and $C$ versus $D$ for width discrimination.

2. In correlated tasks, the two possible stimuli are from the opposite corners of the array, $B$ versus $C$ or $A$ versus $D$. The values of height and width are either negatively correlated ( $B$ versus $C$, a shape-discrimination condition) or positively correlated ( $A$ versus $D$, a sizediscrimination condition).

These first two tasks each involve only two stimuli; the remaining two tasks involve all four:

3 . In selective attention tasks, observers classify the stimuli on the basis of width ( $A$ and $C$ versus $B$ and $D$ ) or height ( $A$ and $B$ versus $C$ and $D$ ). Because the subject attends selectively to just one dimension and tries to filter out the differences between the stimuli on the other, orthogonal, dimension, these are also called "filtering," or "orthogonal," tasks.

4. In the divided attention task, observers must distinguish the negatively correlated stimuli $B$ and $C$ from the positively correlated ones $A$ and $D$. Attention is divided in the sense that subjects must attend to both dimensions. Posner (1964) called this task "condensation."

In Garner's conception, dimensions that are separable are characterized by equal performance in at least the first three of these tasks. The intuition is that the introduction of a second, independent dimension should have no effect on the perception of the first. The canonical example of separable stimuli is the set of circles varying in size and inclination of radius (Shepard, 1964). If the dimensions are integral, on the other hand, the correlated task shows higher performance (a redundancy gain), and the selective task, lower performance (a filtering loss). Garner and Felfoldy's (1970) study of single color patches differing in brightness and saturation exemplifies this pattern. The power of the Garner paradigm lies in its use of multiple tasks - "converging operations"- to distinguish these relations between dimensions. 
Applying the paradigm to rectangle perception, Felfoldy (1974) measured reaction time for an array of stimuli similar to those in Figure 1 and found a filtering loss but no redundancy gain; he concluded that height and width interacted. Dykes and Cooper (1978), who used reaction time, and Dykes (1979), who measured accuracy, found both a filtering loss and a redundancy gain and inferred integrality. What to expect in the correlated task is not clear a priori. In the identification experiments conducted by Monahan and Lockhead (1977) and other investigators, described above, a one-dimensional and a "correlated" condition were also compared, albeit with more than two values per dimension. Lockhead's (1970) perceptual space model implies that pairs of stimuli differing on two dimensions may be more, equally, or less similar than pairs differing on just one. According to this point of view, relative performance on the correlated and baseline tasks depends on the form of the perceptual space and the nature of the redundancy. Support for this hypothesis was obtained by Lockhead and King (1977), who measured speeded classification in the Garner tasks for line pairs like those used by Monahan and Lockhead (1977). A multidimensional representation was derived by applying MDS to dissimilarity judgments, and distances between objects in this space were highly (negatively) correlated with sorting times.

In our experiments, we too made use of the Garner paradigm to study rectangles. In our implementation, physical differences along each dimension were set so that all pairs of stimuli differing on just one dimension were approximately equally discriminable, and accuracy was measured. We interpret our data in terms of an inferred spatial representation in which the distances between elements of the space are measured by the detection-theory sensitivity measure $d^{\prime}$ (not an arbitrary transformation of it).

\section{Integrality and Detection Theory}

Ashby and his colleagues (Ashby \& Maddox, 1994; Ashby \& Townsend, 1986; Kadlec \& Townsend, 1992; Maddox, 1992) have described the central aspects of the Garner paradigm using the constructs of multidimensional detection theory, or generalized recognition theory. Each of the four stimuli is assumed to give rise to a distribution in a perceptual space that, like the stimulus space, has two dimensions. In our application, the distributions are assumed to be equal-variance normal. Each of the four stimuli has an average effect, shown as a square in Figure 2, that corresponds to a location in the space. The distances between the means of the distributions correspond to the discriminabilities of the stimuli. The variability of the distributions is indicated in the figure by circles that join points equally distant (in standarddeviation units) from the mean. In each Garner task, the space is partitioned optimally by the observer into regions corresponding to the possible responses.

The two panels of Figure 2 show two possible arrangements of the four means. In the top panel, the means de-

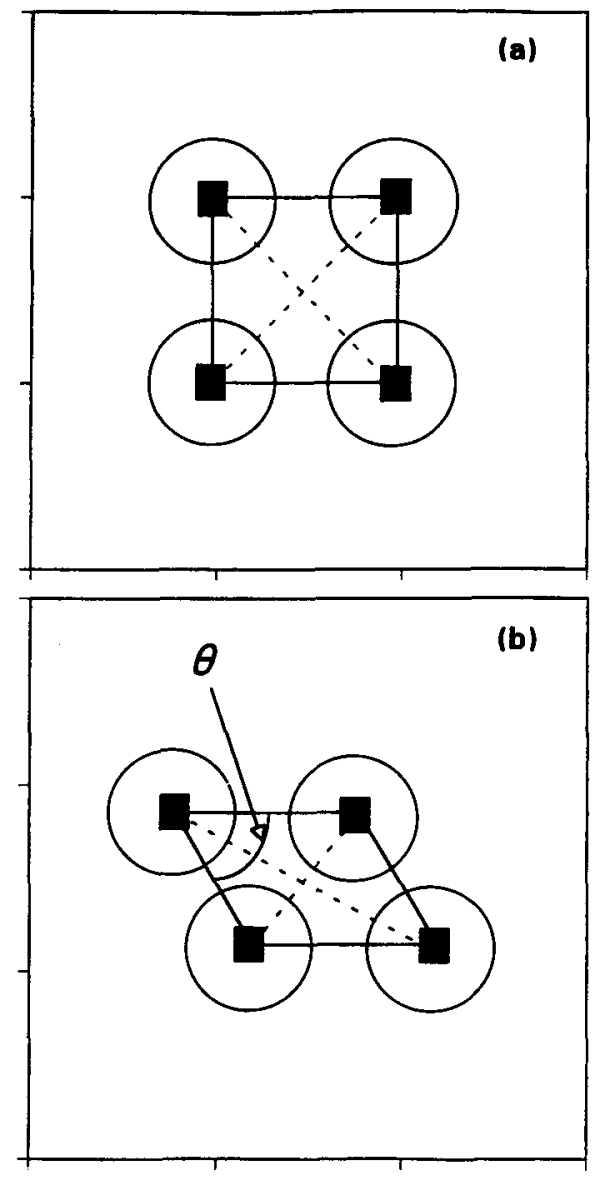

Figure 2. Perceptual space for the Garner paradigm. The figure shows the two dimensions along which observations can vary. Each square is the mean of a bivariate normal distribution; the four squares correspond to the four possible stimuli. The relative likelihood of an observation, given a particular stimulus presentation, could be represented as the height of this distribution in a third dimension; instead, circles indicate values that are equally likely (and equally far from the mean). (a) Perceptually separable dimensions, in which the four means form a rectangle. (b) Mean-integral dimensions, in which the means form a nonrectangular parallelogram.

fine a rectangle, so that the perceptual value of a stimulus on one dimension does not depend on its value on the other. This outcome represents perceptual separability. In the lower panel, the means of the distributions are no longer arranged orthogonally, and the value of a stimulus on one dimension does depend on its value on the other. This violation of independence is described as mean integrality because it arises from a shift in the distribution means relative to the perceptual separability case. ${ }^{1}$

The quantitative implications of this model for Garner paradigm tasks have been described by Ashby and Maddox (1994), Macmillan and Kingston (1995), and Kingston and Macmillan (1995). The key parameter of the model is $\theta$, the angle in the parallelogram in Figure 2, which equals $90^{\circ}$ for perceptually separable dimensions, 
but which is either greater or less than $90^{\circ}$ under mean integrality. Performance in the baseline tasks and one correlated task is sufficient to estimate $\theta$; accuracy levels in the other correlated task, both selective tasks, and the divided task can then be predicted without further assumptions or free parameters. The theory predicts that (1) performance in one correlated task is always better than the average for baseline tasks, and performance in the other may be less than, equal to, or better than in baseline; (2) selective performance is the same as average baseline at $\theta=90^{\circ}$ and poorer otherwise, with the greatest discrepancy at extreme values of $\theta$; and (3) divided performance is always worse than average baseline, by an amount that does not strongly depend on $\theta$. Details of these predictions (and some insight into them) are given in the Results section of Experiment 1.

In the first experiment, we examined rectangles like those in Figure 1. We wished to use the data to infer the perceptual representation and to relate performance in the various Garner tasks quantitatively.

\section{EXPERIMENT 1 Classification of Squares and Rectangles}

\section{Method}

Subjects. The 8 subjects, 4 men and 4 women, were graduate and undergraduate students at Brooklyn College and ranged in age from 20 to 35 . All reported normal (or corrected-to-normal) vision and were naive regarding the purposes of the experiment. They were paid an hourly wage.

Viewing conditions. The subjects sat individually in a soundattenuated, dimly lit room. They faced a computer monitor $0.75 \mathrm{~m}$ from them; the distance was maintained by use of a chinrest.

Stimuli. The screen, which measured $243 \mathrm{~mm}$ horizontally by $187 \mathrm{~mm}$ vertically, displayed $640 \times 350$ pixels. The stimuli consisted of straight dark lines on a white background. There were two stimulus sets, squares and rectangles. In the square set, one stimulus (stimulus $A$ in Figure 1) had horizontal and vertical lines with a length of $49.7 \mathrm{~mm}$. In the rectangle set, stimulus $A$ had a horizontal line with a length of $52.4 \mathrm{~mm}$ and a vertical line with a length of $26.2 \mathrm{~mm}$. The other stimuli in both sets were formed by increasing the horizontal, vertical, or both lines by small amounts $\Delta_{\mathrm{H}}$ and $\Delta_{\mathrm{V}}$.

The stimuli were presented in the approximate center of the screen. To guard against judgments based on distances to the edge of the screen rather than the object itself, the exact location of the stimulus varied randomly from trial to trial by a maximum of $9.9 \mathrm{~mm}$ horizontally and $10.2 \mathrm{~mm}$ vertically.

The values of $\Delta_{\mathrm{H}}$ and $\Delta_{\mathrm{V}}$ were chosen individually for each subject so that, if possible, performance was above chance but less than perfect in all conditions. Because observers were able to resolve differences of only a pixel or two in the vertical direction, a limited set of values that could be used in both dimensions was available. For 3 of the 8 subjects, performance in one of the correlated tasks was at ceiling using values of $\Delta_{\mathrm{H}}$ and $\Delta_{\mathrm{V}}$ from the other tasks; in these cases, smaller stimulus differences were used in both correlated tasks. Table 1 gives the values of $\Delta_{\mathrm{H}}$ and $\Delta_{\mathrm{V}}$ for all subjects.

Procedure. On each trial, a single stimulus was presented and remained on the screen until the subject responded. Each task required the subject to categorize the stimuli as one of two types, using a rating response $(1=$ sure it was type $I, 2=$ probably type $I, \ldots, 6=$ sure it was type $I I)$. As soon as the response was made on the computer keyboard, a tone provided feedback about correctness. (For this purpose, Responses 1-3 were considered correct for
Table 1

Stimulus Parameters, Experiment 1

\begin{tabular}{|c|c|c|c|c|}
\hline \multirow[b]{2}{*}{ Shape } & \multirow[b]{2}{*}{ Subject } & \multirow[b]{2}{*}{ Task } & \multicolumn{2}{|c|}{ Stimulus Differences (\%) } \\
\hline & & & $\Delta_{\mathrm{H}}$ & $\Delta_{\mathrm{V}}$ \\
\hline \multirow[t]{6}{*}{ Square } & 1 & All & 2.29 & 2.15 \\
\hline & 2 & All & 3.05 & 3.23 \\
\hline & 3 & Correlated & 2.29 & 2.15 \\
\hline & & Other & 3.05 & 3.23 \\
\hline & 4 & Correlated & 2.29 & 2.15 \\
\hline & & Other & 3.05 & 3.23 \\
\hline \multirow[t]{5}{*}{ Rectangle } & 5 & Correlated & 2.17 & 2.04 \\
\hline & & Other & 4.35 & 4.08 \\
\hline & 6 & All & 2.17 & 2.04 \\
\hline & 7 & All & 2.17 & 2.04 \\
\hline & 8 & All & 4.35 & 4.08 \\
\hline
\end{tabular}

one stimulus subset, Responses 4-6 for the other.) As a memory aid, subjects were given a piece of paper illustrating the stimuli (using exaggerated values of $\Delta_{H}$ and $\Delta_{V}$, as in Figure 1). The large number of different tasks made this a useful reference.

The experiment was conducted in 1-h segments, with rest breaks. The subjects received enough training (between 4 and $8 \mathrm{~h}$ ) so that, in the judgment of the experimenter, performance had reached an asymptote. The experiment proper comprised the nine tasks of the Garner paradigm - four baseline, two correlated, two selective, and one divided-administered in a different quasi-random order to each observer. Within a condition, there were four blocks of trials: The first (consisting of 20 trials) was practice, and the other three ( 55 trials each) began with 5 practice trials. Except for the baseline conditions, the subject experienced each condition twice, on different days. Three or four conditions, a total of 555 or 740 trials, usually made up a 1 -h session. The subjects served for between 16 and $25 \mathrm{~h}$ overall (including practice sessions).

\section{Results}

Detection theory analysis. The rating data were used to estimate the best-fitting ROC curves, separately for each observer, using the Dorfman and Alf (1969) program. All trials in a given condition contributed to a single ROC, so that, for example, the curves for the correlated task in which stimuli $A$ and $D$ were discriminated are based on 300 trials. As our measure of accuracy, we chose $d_{a}$, a transform of the area under the best-fitting normal-distribution ROC curve that estimates the distance between the means of the two distributions, in units of the root-mean-square average standard deviation. (See Swets \& Pickett, 1982, and Macmillan \& Creelman, 1991, for discussion of this statistic.) The slopes of the ROCs did not deviate significantly or systematically from 1.0, and we do not report them.

This type of detection theory analysis assumes that each of two stimulus classes gives rise to a single normal distribution on a decision axis. For the baseline and correlated tasks, the analysis is appropriate; each condition involves two stimuli that may be considered to project onto an axis drawn through their means in Figure 2. But the use of four stimuli in the selective and divided tasks leads, by hypothesis, to distributions whose locations differ two-dimensionally, so these tasks cannot be analyzed properly by the two-stimulus model. We did so anyway in order to obtain comparable performance mea- 
sures in all conditions, but the resulting values are heuristic, not estimates of the theoretical parameters of any detection-theoretic model. More sophisticated treatment of the four-stimulus conditions will be presented later.

Recall that in the correlated task the size of the stimulus differences was reduced for some subjects. The distance-measure characteristic of $d_{a}$ provides a simple way to correct for this discrepancy. Consider Subject 3, for whom $\Delta_{\mathrm{H}}=3.05$ and $\Delta_{\mathrm{V}}=3.23$ for most tasks, but for whom $\Delta_{\mathrm{H}}=2.29$ and $\Delta_{\mathrm{V}}=2.15$ for the correlated tasks. The observed $d_{a}$ in each correlated task was corrected by the ratio of the Pythagorean distances for the two stimulus sets; that is, it was multiplied by $\left[\left(3.05^{2}+3.23^{2}\right) /\right.$ $\left.\left(2.29^{2}+2.15^{2}\right)\right]^{1 / 2}=1.41$.

Summary statistics and traditional analysis. The average $d_{a}$ and its standard error (across subjects) for each condition in Experiment 1 are given in Table 2; for the correlated tasks, it is the "corrected" values that appear. Squares and rectangles are treated separately, so each statistic is based on 4 observers. The pattern of results is the same for squares and rectangles: One correlated task is easier than either baseline; the other, harder. Selective performance is poorer than baseline, and divided performance is poorer yet.

We evaluated the statistical reliability of our results as follows. For a single observer, the standard error associated with $d^{\prime}$ can be calculated from an approximation due to Gourevitch and Galanter (1967). To apply this method to rating data, we treated values of $d_{a}$ as though they were values of $d$ 'produced by an unbiased observer. To extend the analysis to the average of the 4 subjects, we averaged the $d^{\prime}$ values; the variance associated with this estimate equals $1 / 16$ of the sum of the variances associated with each subject.

Gourevitch and Galanter's (1967) analysis applies only to the cases of one or two conditions, whereas the present experiment had nine (counting the two width baseline and two height baseline conditions separately). Marascuilo (1970) extended the method to experiments of this type; applying his technique to the data, we found highly significant differences among the nine means $\left[\chi^{2}(8)=227.9\right.$ for squares, 220.7 for rectangles, $p<$ .001 in both cases]. Marascuilo also presented a technique for evaluating contrasts, and we examined seven comparisons: (1) there was no reliable difference between the two horizontal baseline tasks (2) or the two

Table 2

$d_{a}$ Values for Each Task in Experiment 1

\begin{tabular}{lccccc}
\hline & \multicolumn{2}{c}{ Squares } & & \multicolumn{2}{c}{ Rectangles } \\
\cline { 2 - 3 } \cline { 5 - 6 } \multicolumn{1}{c}{ Task } & $M$ & $S E$ & & $M$ & $S E$ \\
\hline Baseline (width) & 1.45 & 0.35 & & 1.70 & 0.42 \\
Baseline (height) & 1.55 & 0.27 & & 1.36 & 0.36 \\
Correlated (size) & 1.20 & 0.44 & & 0.90 & 0.14 \\
Correlated (shape) & 2.75 & 0.66 & & 2.57 & 0.84 \\
Selective (width) & 1.14 & 0.16 & & 1.55 & 0.33 \\
Selective (height) & 1.24 & 0.13 & & 1.24 & 0.14 \\
Divided & 0.70 & 0.37 & & 0.61 & 0.27 \\
\hline
\end{tabular}

vertical ones, justifying our parallelogram assumption; (3) average correlated performance exceeded average baseline $(p<.05)$ - that is, there was a redundancy gain; (4) average selective performance was less than average baseline ( $p<.05$ for squares, $p=.06$ for rectangles $)-$ that is, there was a filtering loss; (5) selective performance on a single dimension was reliably lower than on the corresponding baseline tasks in only one of the four cases, height discrimination in the rectangle condition; (6) the $B C$ correlated task (shape discrimination) was performed more accurately than the $A D$ task (size) ( $p<$ .05 ); and (7) the divided task was more difficult than average baseline $(p<.05)$. The statistical pattern of results was thus essentially the same for squares and rectangles.

There was not enough power to conduct the entire analysis on single subjects, but the individual subject data do confirm the analysis of the average results. Seven of the 8 subjects showed a reliable asymmetry between the correlated tasks, 4 ( 2 for each stimulus set) showed significant correlated gains, and 4 ( 2 for each set) showed significant drops in the divided condition. No subject was reliably different for two baseline tasks on the same dimension (AB vs. $C D$ or $A C$ vs. $B D$ ).

A traditional Garner analysis might reach either of two conclusions, depending on whether only the average of the two baseline and two correlated tasks is considered, or whether these are distinguished. The average data show that correlated performance is superior to baseline $\left(d_{a}=1.98 \mathrm{vs} .1 .50\right.$ for squares, $1.74 \mathrm{vs} .1 .53$ for rectangles), an overall redundancy gain; combined with the filtering loss, this pattern leads to the diagnosis of integrality. A more detailed analysis would note that one correlated task is better, the other worse, than average baseline; combined with the superiority of selective over divided performance (which rules out configurality as a syndrome), this pattern is what Melara and O'Brien (1987) have termed correspondence.

Parallelogram representations. According to our models, the correlated tasks are the key to distinguishing between the perceptually separable and mean-integral cases. If the two correlated conditions yield equal performance that is slightly better than baseline, the stimulus dimensions are perceptually separable. If, as in our data, one correlated task is easier than the other, the stimuli are mean-integral, the extent of mean integrality corresponding to the acute angle $\theta$ of the best-fitting parallelogram (see Figure 2). Fitting parallelograms is appropriate whenever performance for the two baseline tasks on the same dimension is equal; the data showed no systematic violations of this simplification.

We fit our model to the data as follows. Using the baseline $d^{\prime}$ values, a series of baseline parallelograms was formed, with the angle $\theta$ between segments $A B$ and $A C$ ranging from $0^{\circ}$ to $180^{\circ}$. Segments of length equal to the correlated $d^{\prime}$ values were drawn on the diagonals of the parallelograms; these were in general either too long or too short to reach the corners. For each angle $\theta$, a compromise parallelogram was formed from points be- 
tween the points on the baseline parallelogram and the points at the end of the correlated segments, and a rootmean-square error ( $R M S E)$ for this parallelogram was found. To calculate $R M S E$, both observed $d^{\prime}$ and that predicted from the parallelogram were converted to the percent correct obtainable by an unbiased observer. From the compromise parallelogram for which the RMSE was minimal, we took our estimate of the angle $\theta$. Note that the estimate depended only on the outcomes of the twostimulus tasks.

We performed this analysis on the data of each subject separately, and on the average data of each group. The average data, shown in Figure 3, reveal mean integrality for both stimulus sets. For the squares, $\theta$ was $133^{\circ}$; for the rectangles, $144^{\circ}$. The parallelograms pro-
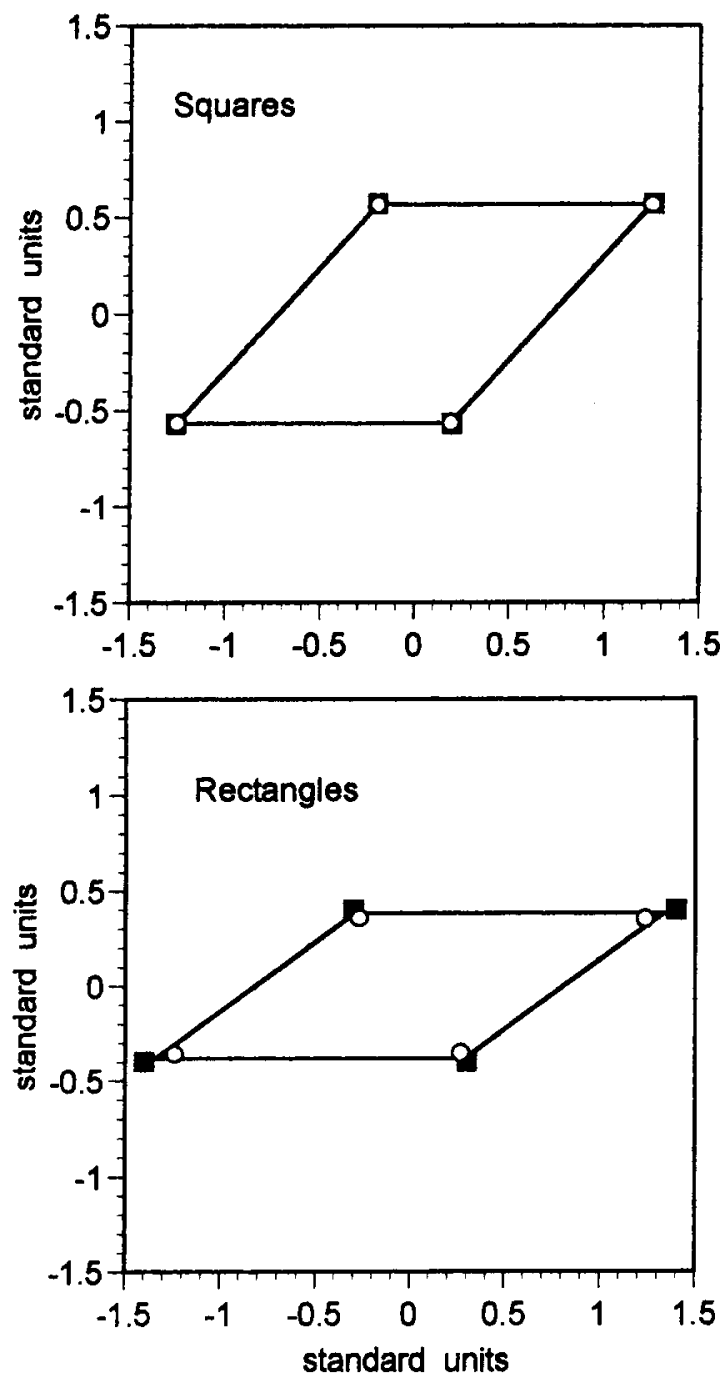

Figure 3. Parallelogram representations of the results of Experiment 1. Distances between squares are observed $d_{a}$ values for baseline tasks; distances between circles, the observed $d_{a}$ values for correlated tasks. The parallelogram is drawn to minimize the squared difference of the points from the figure, in units of proportion correct $\left(\operatorname{not} d_{a}\right)$.

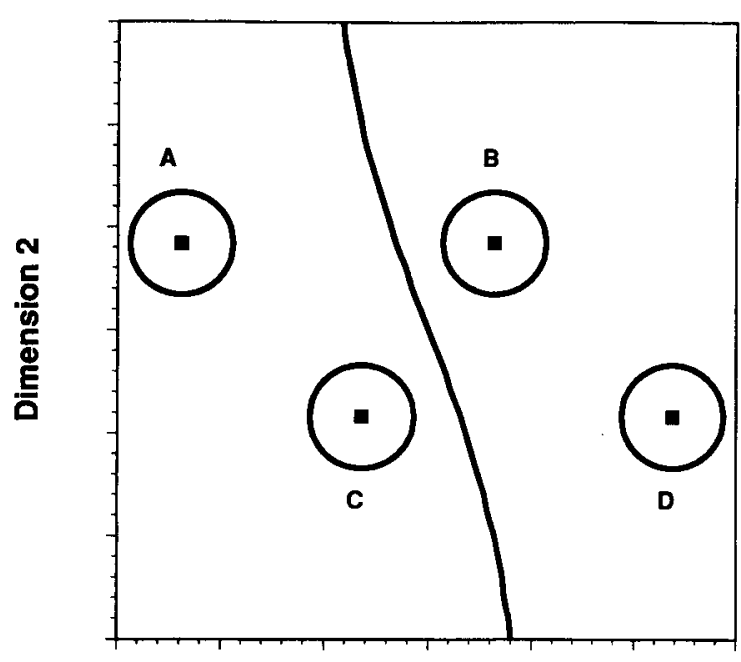

Dimension 1

Figure 4. Optimal decision rule for the selective attention task. Each circle represents a distribution in perceptual space, as in Figure 2. The curves define the optimal decision rule: The observer makes one response for points to the left of the curve, the other for points to the right.

vided an excellent fit to the average data: The RMSE is 0.3 percentage points for squares, 1.2 for rectangles. Individual subject data were consistent with this overall picture. For squares, $\theta$ ranged from $119^{\circ}$ to $154^{\circ}, R M S E$ from 0.8 to 5.1 percentage points; for rectangles, $\theta$ ranged from $116^{\circ}$ to $156^{\circ}, R M S E$ from 0.1 to 4.5 .

Selective attention. Performance in both selective attention tasks can be predicted without free parameters from the assumption that observers adopt an optimal decision rule. In the perceptually separable, $\theta=90^{\circ}$ case (Figure 2a), an observer trying to classify stimuli $A$ and $B$ into one category, $C$ and $D$ into the other, does best by using a horizontal criterion line. Performance is the same as in the $A$ versus $C$ baseline task. Figure 4 shows decision bounds for the mean-integral situation $\left(\theta<90^{\circ}\right.$ or $\theta>90^{\circ}$ ). Predicted performance declines with $\theta$; intuitively, this is because the distributions due to $B$ and $C$, which belong to different categories, are increasingly close together. Detailed predictions are given in Macmillan and Kingston (1995); Maddox (1992) has shown similar results for a slightly different criterion curve, and for equal-sided parallelograms only.

The average data are shown in Table 3. Observed proportion correct was adjusted to the level that would be expected in the absence of bias. To obtain predicted values, we calculated the optimal criterion bound for the parallelogram inferred earlier and predicted performance for each subject and stimulus condition. Observed performance fell short of predicted by an average of 3.2 percentage points for squares, but actually exceeded predictions by an average of 1.4 points for rectangles, for an average discrepancy of 0.9 points. Overall, the model predicted a filtering loss of three points, and the ob- 
Table 3

Predicted and Observed Percent Correct for Selective and Divided Attention in Experiment 1

\begin{tabular}{lccccccc}
\hline & \multicolumn{3}{c}{ Squares } & & \multicolumn{3}{c}{ Rectangles } \\
\cline { 2 - 4 } \cline { 6 - 7 } \multicolumn{1}{c}{ Task } & Predicted & Observed & Difference & & Predicted & Observed & Difference \\
\hline Selective (width) & 75.0 & 71.5 & 3.5 & & 76.5 & 77.1 & -0.6 \\
Selective (height) & 76.0 & 73.1 & 2.9 & & 70.9 & 73.1 & -2.2 \\
Divided & 67.3 & 62.9 & 4.4 & & 66.8 & 61.7 & 5.1 \\
\hline
\end{tabular}

served loss was four. Mean integrality, as inferred from the two-stimulus conditions, predicts accuracy in the selective condition quite well.

Divided attention. In the perceptually separable, $\theta=$ $90^{\circ}$ case, an observer trying to classify stimuli $A$ and $D$ into one category, $B$ and $C$ into the other, does best by dividing the perceptual space into quadrants and assigning events in opposite quadrants to the same response. Performance is substantially worse than at baseline. (When all four baselines are equal, the task is equivalent to same-different discrimination; expected performance in this task, with this rule, has been calculated by Macmillan \& Creelman, 1991.) Figure 5 shows the optimal decision rule for the mean-integral case. Predicted performance is substantially lower than baseline and is little affected by $\theta$. Detailed calculations are presented in Macmillan and Kingston (1995).

The data are summarized in Table 3. Predicted values were again calculated, using the inferred parallelograms and optimal decision bounds for each subject individually. Observed performance averaged 4.7 percentage points less than predicted. This is a larger discrepancy than in selective attention, but the decline, in comparison with baseline, is also greater: The model predicts 10.7 points of the observed 15.4-point decline.

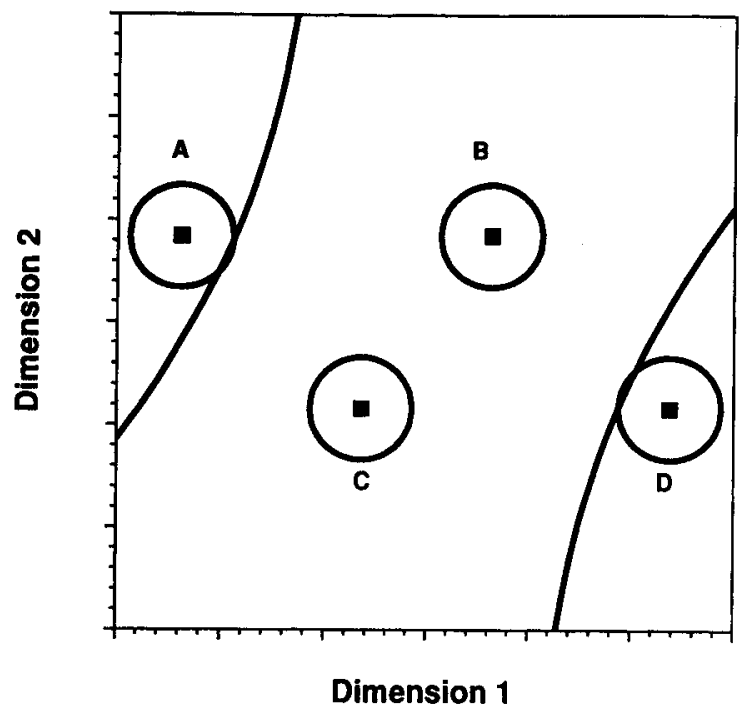

Figure 5. Optimal decision rule for the divided attention task. As in Figure 4, except that the observer now makes one response for points between the two curves, the other for points outside them.
Overall fit of the model. To evaluate the fit of the parallelograms to the data more systematically, we conducted a series of $\chi^{2}$ tests. Each $d^{\prime}$ was converted back to a proportion correct, assuming no response bias, and the numbers of correct and incorrect responses corresponding to each proportion were found. These were compared, using $\chi^{2}$, to the response rates expected from the inferred parallelogram. This procedure was done four times: using just the two-stimulus tasks, two-stimulus and divided attention, two-stimulus and selective attention, and all tasks. The $d f$ associated with the two-stimulus tasks alone is one: Barring a violation of the triangle inequality, a parallelogram can be exactly determined by the two baseline $d^{\prime}$ values plus a single correlated $d^{\prime}$, and the second correlated $d^{\prime}$ contributes a degree of freedom. The other tests involve two, three, and four $d f$ respectively.

With 4 subjects in each of two conditions, there are eight tests for each of the four sets of conditions. The value of $\chi^{2}$ was too small to reject the null hypothesis (that the best-fitting parallelogram generated the data) in 4 of 8 cases for the baseline-only test, and in 3,5 , and 3 of 8 cases for the other three tests. Thus, 15 of 32 cases provided acceptable fits. These cases were not equally distributed between the two stimulus conditions, however: 13 of 16 fits were obtained with the rectangle stimuli, and only 2 of 16 with the squares. We have no ready explanation for this discrepancy, but many of the "poor" fits are not very poor: If the criterion for a fit is relaxed to $p>.01$, an additional 6 square conditions provide acceptable fits. Because the subjects for the two shapes were different, it is not possible to determine whether the difference had to do with the shape being perceived or the individuals doing the perceiving.

\section{Discussion}

In agreement with previous researchers, we find that the height and width of rectangles interact in perception. The results follow a pattern of mean integrality: There is a filtering loss, and performance on the two correlated tasks is unequal. The mean-integral model provided an excellent fit to the two-stimulus data. Selective performance was also well predicted, falling an average of one percentage point below its expected level. The larger discrepancy in divided attention (though modest compared with the divided-baseline difference) suggests that this condition may lead to changes in the underlying distributions or may force observers to adopt nonoptimal deci- 
sion rules (see Maddox \& Ashby, 1996, for an example of such an effect).

Our conclusions about representation are similar to those of Weintraub (1971), who noted that if the perceptual space for his data were constructed from orthogonal dimensions corresponding to height and width, the distributions associated with the rectangles would be elliptical, in such a way that the confusability of a stimulus would be greater with an adjacent one differing in size than with an adjacent one differing in shape.

A geometric interpretation of the mean integrality of height and width draws on Figure 3. Consider a line drawn in the plane of the figure connecting the means of the distributions corresponding to stimuli $B$ and $C$. This line defines a shape dimension, and judgments of size are orthogonal to it. The perceptual space can be interpreted as having two dimensions, size and shape, of very unequal sensitivity. These dimensions may be independent, although our data do not speak directly to this issue (see the General Discussion). If the space is defined by height and width, on the other hand, the sensitivities are about equal, but the dimensions are not independent.

We used both squares and rectangles in this experiment because we imagined that squares might represent a special case. Width discrimination might be easier for squares than for rectangles, because the figure's height would be available as a standard. The data do not support this speculation: Width thresholds were $1.99 \mathrm{~mm}$ for squares and $1.87 \mathrm{~mm}$ for rectangles. This small difference is not in the right direction for the hypothesis, and it is inconsistent with Weber's law. In our experimental situation, it is just the difference in the widths of the figures that matters, not the total width or its relation to height.

\section{EXPERIMENT 2 Classification of Line Pairs}

A question raised by Experiment 1 is, How do height and width perception of rectangles combine to produce area or shape judgments? A simple hypothesis is that they combine independently; for discrimination data, this is equivalent to the idea that $d^{\prime}$ values combine in a Pythagorean fashion. We have seen that this is not correct - if it were, height and width would have been perceptually separable - but that a generalized form of the hypothesis, in which better than independent performance for shape is balanced with worse than independent performance for size, does hold.

Another approach to the combination question is to consider stimuli that are rectangle segments, rather than complete rectangles. In the second experiment, we used line pairs, horizontal and vertical line segments that formed the upper left half of the rectangles in Experiment 1 . Such stimuli were used by Ashby and Gott (1988). We wished to know whether these stimuli would show the same pattern of perceptual interaction as did rectangles themselves. We also hoped to use the comparison between the line pairs and the rectangles to ask
Table 4

Stimulus Parameters, Experiment 2

\begin{tabular}{|c|c|c|c|c|}
\hline \multirow[b]{2}{*}{ Shape } & \multirow[b]{2}{*}{ Subject } & \multirow[b]{2}{*}{ Task } & \multicolumn{2}{|c|}{ Stimulus Differences (\%) } \\
\hline & & & $\Delta_{\mathrm{H}}$ & $\Delta_{\mathrm{V}}$ \\
\hline \multirow[t]{6}{*}{ Equal sides } & 1 & Correlated & 3.05 & 3.23 \\
\hline & & Other & 4.58 & 4.30 \\
\hline & 2 & All & 5.34 & 5.38 \\
\hline & 3 & Correlated & 3.05 & 3.23 \\
\hline & & Other & 4.58 & 4.30 \\
\hline & 4 & All & 3.05 & 3.23 \\
\hline \multirow[t]{5}{*}{ Unequal sides } & 5 & All & 2.17 & 2.04 \\
\hline & 6 & All & 2.17 & 2.04 \\
\hline & 7 & Correlated & 2.17 & 2.04 \\
\hline & & Other & 4.35 & 4.08 \\
\hline & 8 & All & 4.35 & 4.08 \\
\hline
\end{tabular}

questions about how the perception of rectangles depended on the perception of their parts.

\section{Method}

The subjects from Experiment 1 also served in Experiment 2. (From the subjects' point of view, it was a single experiment, and they were not debriefed until all conditions had been completed.) The viewing conditions and procedures were the same as in Experiment 1 . The stimuli were the left and top sides of the stimuli from Experiment 1, joined at the corner. Thus, subjects who had judged squares in Experiment 1 judged equal-length line pairs in Experiment 2 , whereas those who had judged rectangles saw line pairs in which the horizontal segment was twice the length of the vertical segment. Other aspects of stimulus presentation were identical. Table 4 gives the values of $\Delta_{\mathrm{H}}$ and $\Delta_{\mathrm{V}}$ for each subject in each condition.

\section{Results}

Summary statistics and traditional analysis. The average $d_{a}$ and its standard error for each condition in Experiment 2 are given in Table 5; for the correlated tasks, it is the "corrected" values that appear. The pattern of results for equal- and unequal-length line pairs is the same, and it is the same as for squares and rectangles: One correlated task is easier than either baseline, the other harder. Selective performance is poorer than baseline, and divided performance is poorer yet.

Considering only the average data for equal sides, correlated performance is superior to baseline $\left(d_{a}=2.08\right.$ vs. 1.58 ), an overall redundancy gain; combined with a filtering loss of 0.26 , this pattern leads to the diagnosis of integrality. For the unequal sides, the redundancy gain (1.28 vs. 1.02 ) is small but reliable, the filtering loss (0.04) small and unreliable; it is unclear which of the

Table 5

$d_{a}$ Values for Each Task in Experiment 2

\begin{tabular}{lccccc}
\hline \multirow{2}{*}{\multicolumn{1}{c}{ Task }} & \multicolumn{2}{c}{ Equal sides } & & \multicolumn{2}{c}{ Unequal sides } \\
\cline { 2 - 3 } \cline { 6 - 6 } & $M$ & $S E$ & & $M$ & $S E$ \\
\hline Baseline (width) & 1.68 & 0.06 & & 1.19 & 0.13 \\
Baseline (height) & 1.49 & 0.27 & & 0.84 & 0.14 \\
Correlated (size) & 1.55 & 0.27 & & 0.93 & 0.09 \\
Correlated (shape) & 2.62 & 0.39 & & 1.64 & 0.19 \\
Selective (width) & 1.42 & 0.12 & & 1.06 & 0.14 \\
Selective (height) & 1.26 & 0.19 & & 0.91 & 0.15 \\
Divided & 0.81 & 0.18 & & 0.41 & 0.21 \\
\hline
\end{tabular}



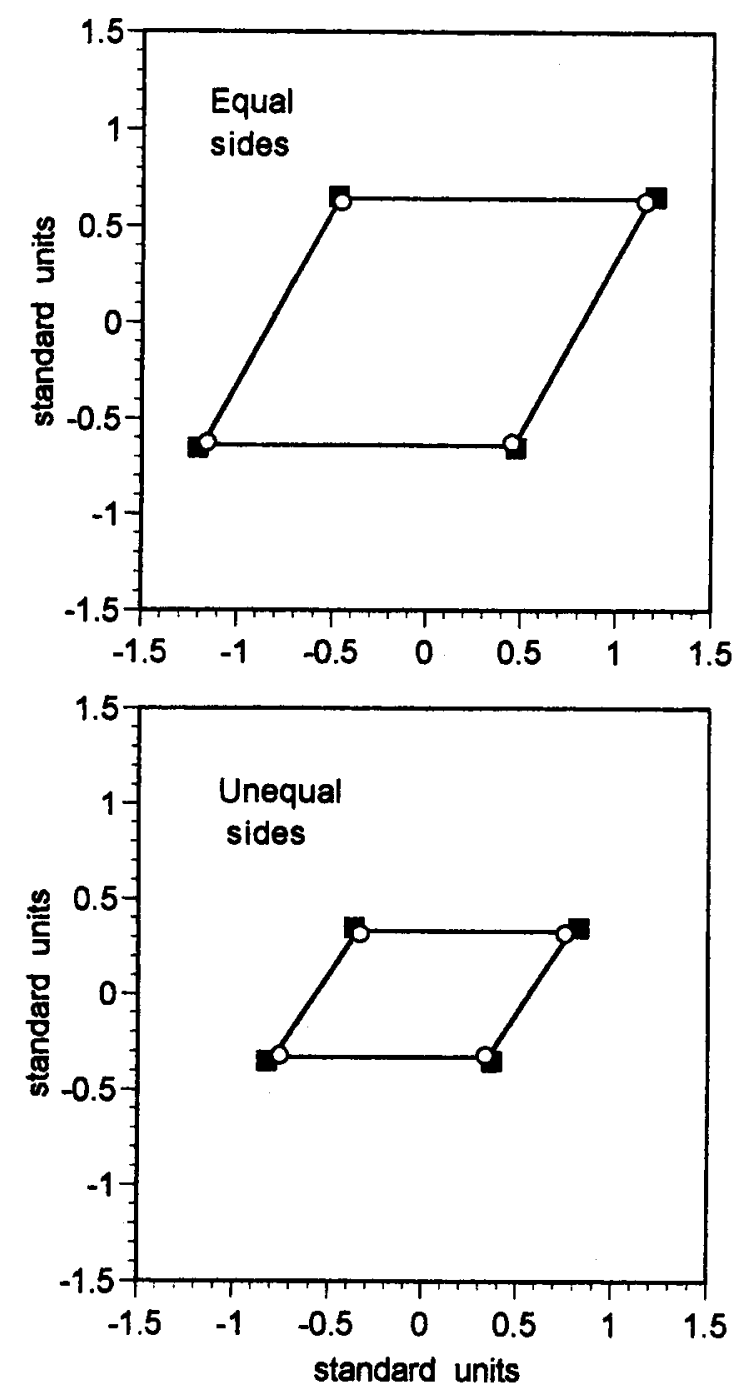

Figure 6. Parallelogram representations of the results of Experiment 2 (see Figure 3 for details).

accepted patterns this most closely fits. As in Experiment 1 , one correlated task (shape) is better than the other (size), but size discrimination is not worse than average baseline.

Parallelogram representations. We again found the best-fitting parallelogram in perceptual space from the data of each subject separately, and from the average data of each group. The average data, shown in Figure 6, indicate mean integrality. For equal-sided figures, $\theta$ was $119^{\circ}$; for the unequal-sided ones, $123^{\circ}$. The parallelo- grams provided an excellent fit to the data: The RMSE is 0.4 percentage points for equal sides, 0.8 for unequal ones. Individual subject data were consistent with this overall picture. For equal sides, $\theta$ ranged from $94^{\circ}$ to $139^{\circ}$, RMSE from 0.5 to 2.5 percentage points; for unequal sides, $\theta$ ranged from $96^{\circ}$ to $145^{\circ}, R M S E$ from less than 0.1 to 3.0 points.

The average results for selective and divided attention are shown in Table 6. Observed selective performance fell short of predicted by an average of three percentage points for equal sides, and slightly exceeded predictions (by an average of one point) for unequal sides; the average discrepancy across conditions is thus one point. Observed divided performance averaged only 0.6 percentage points less than predicted. The mean-integral parallelogram representations predict accuracy in the four-stimulus conditions very well.

Parallelogram fits to the data were acceptable for the two-stimulus conditions in 7 of 8 cases, for two-stimulus plus divided also in 7 cases, for two-stimulus plus selective in 4 cases, and for all conditions in 4 cases. Altogether, 22 of 32 cases provided good fits. As in Experiment 1 , these were unequally divided between the two stimulus conditions, with 14 of 16 fits being obtained with unequal line segments and 8 of 16 with equal line segments. The confound between subjects and stimulus conditions, noted in Experiment 1, was true here as well. The apparent improvement in the number of good fits, in comparison with what was found in Experiment 1, suggests no ready explanation.

\section{Discussion}

Experiments 1 and 2 differed only in that closed rectangular figures were used as stimuli in the first, whereas line pairs joined at a corner were used in the second. The inferred representations of the stimulus sets were quite similar: parallelograms with interior angles of about $138^{\circ}$ for closed figures and $121^{\circ}$ for line pairs. The absolute levels of performance differed, however, with rectangle discrimination being in general somewhat better than line-pair discrimination. For length discrimination (baseline conditions), $d^{\prime}$ was better for rectangles than for line pairs by a factor of 1.23 ; for size discrimination (positively correlated pairs), by a factor of 1.07 ; and for shape discrimination (negatively correlated pairs), by a factor of 1.39. Adding a second pair of lines maximally improved performance in shape discrimination, did so to some degree in length discrimination, and was rather unhelpful in size discrimination. In this section, we will explore a simple explanation for this pattern of results.

Table 6

Predicted and Observed Percent Correct for Selective and

Divided Attention in Experiment 2

\begin{tabular}{lccccccc}
\hline & \multicolumn{3}{c}{ Equal sides } & & \multicolumn{3}{c}{ Unequal sides } \\
\cline { 2 - 4 } Task & Predicted & Observed & Difference & & Predicted & Observed & Difference \\
\hline Selective (width) & 78.7 & 75.9 & 2.9 & & 71.0 & 70.0 & 1.0 \\
Selective (height) & 75.9 & 73.4 & 2.5 & & 64.6 & 67.4 & -2.8 \\
Divided & 67.1 & 65.6 & 1.5 & & 57.7 & 58.0 & -0.3 \\
\hline
\end{tabular}


Suppose that the observer in rectangle discrimination makes two observations about the stimulus, one based on the upper and left segments, the other based on the lower and right ones. These two observations both provide information about the discrimination being measured, thus providing the observer with two samples of data relevant to a decision. The first of these observations is exactly the same as that available to the observer in line-pair discrimination; the second is formally equivalent. Having two samples rather than one could allow better performance; how much better depends on whether the observations are independent or correlated.

A simple detection-theoretic model can quantify this relation. Consider an observer who obtains $d_{2}^{\prime}$ for a baseline task for two-segment stimuli (i.e., line pairs). In the perceptual space, the shorter and longer of the two segments have a mean distance of $d_{2}^{\prime}$ and unit variance. Suppose this observer performs in the same baseline task with four-segment stimuli (i.e., rectangles) by adding observations for two line pairs. The mean difference is now $2 d_{2}^{\prime}$, and the variance is the sum of the variances plus $2 r$ times the product of the standard deviations, or $2+2 r$. Thus sensitivity in the four-segment case, $d_{4}^{\prime}$, is related to sensitivity in the two-segment case by

$$
d_{4}^{\prime}=\frac{2 d_{2}^{\prime}}{\sqrt{2(1+r)}} \text {. }
$$

If the two observations are independent $(r=0)$, performance will improve by $\sqrt{2}$, whereas if they are completely correlated, it will not improve at all. All the observed $d^{\prime}$ ratios are within the interval from 1.0 to 1.41 , as this model implies.

Equation 1 can be solved for $r$ and applied to the baseline and correlated conditions. (Recall that we cannot directly estimate $d^{\prime}$ values for the selective and divided attention tasks.) The implied correlations are .24 for length discrimination, .75 for size discrimination, and .03 for shape discrimination. Thus, if observers attend to all relevant segments, it appears that their observation of parallel segments is quite redundant for size discrimination, quite independent for shape, and intermediate for length.

Alternatively, observers may not attend to all segments in all conditions. The obtained pattern of results would arise if all four segments were necessary to make judgments about shape, but only two to make judgments about size, with length judgments being based sometimes on two and sometimes on four segments.

\section{GENERAL DISCUSSION}

These experiments invite conclusions about three substantive issues: the nature of the perceptual representation for rectangles, the presence or absence of interaction between perceptual dimensions, and whether perception of rectangles is understood better as holistic or in terms of parts. We will discuss each of these questions in turn and also comment on the approach that led us to our conclusions.

\section{The Perceptual Representation}

Treating the discrimination $d^{\prime}$ values from the various Garner conditions as distances in a perceptual space, we inferred a geometric representation in two-dimensional space for each of our rectangle and line-pair stimulus sets. Because the two baseline tasks on the same dimension were always (statistically) equally difficult, we concluded in each of four cases that the points formed a parallelogram. The parallelograms were not rectangular; rather, one interior angle approximated $130^{\circ}$. The asymmetry arose because observers discriminated shape (stimulus $B$ vs. $C$ ) much more easily than size (stimulus $A$ vs. $D$ ).

In general, this geometrical model describes the data well. ${ }^{2}$ The two-stimulus tasks (length, size, and shape discrimination) can be characterized by mean-integral representations in which the average $R M S E$ is only 0.7 percentage points. In the selective and divided attention tasks, observed performance fell below predicted by an average of 0.9 and 2.7 points, respectively. Statistically, the model provides acceptable fits to the individual data from two-stimulus conditions in 11 of 16 cases, and to the complete data set (including the attention-demanding conditions) in 7 of 16 .

The parallelogram representation is consistent with conclusions reached by investigators using identification designs. Weintraub's (1971) identification study led him to conclude that decisional variability was greater along the size axis than along the shape axis; he pointed out (pp. 6-7) that this description is equivalent to tilting the axes or adjusting the mean locations. The results are also in agreement with the general principle put forth by Monahan and Lockhead (1977) that psychophysical performance with rectangle sets depends on the perceptual spacing of the stimuli.

It is more difficult to compare our results with those obtained by using MDS, because of the disagreements among different MDS studies (see the introduction). However, our representations are qualitatively similar to those obtained by Lockhead and King (1977) for line pairs. We believe that the present discrimination methodology offers a valuable complement to MDS: Our distance measures may be less influenced by higher level cognitive variables (see, e.g., Krumhansl, 1978) but are limited by the need to use stimuli that are closely spaced. One way to overcome the latter disadvantage is to perform identification experiments with larger stimulus sets; methods for interpreting such experiments in terms of generalized recognition theory have been developed by Kadlec (1995; Kadlec \& Townsend, 1992).

\section{Interactions Between Dimensions}

Because orthogonal dimensions indicate independence in spatial terms, our finding that the perceptual 
height and width axes are not orthogonal shows that height and width interact in perception. The particular form of the interaction, mean integrality, is one in which a change in the level of one variable (say, width) affects the mean perceptual location in the other (say, height), but not its variability.

Previous researchers using the Garner paradigm (Dykes, 1979; Dykes \& Cooper, 1978; Felfoldy, 1974) have also concluded that height and width interact, but the grounds for this conclusion have been somewhat different. The diagnosis of interaction in these studies was based on the observation of a filtering loss and (in the cases of Dykes and of Dykes and Cooper) a correlated gain. In our data, most subjects showed a reliable filtering loss and correlated gain, implying "integrality" by traditional criteria (Garner, 1974). In this respect, we are in agreement with these earlier studies.

Most of our observers also showed a reliable difference between the two correlated tasks; in combination with the other results, this is the hallmark of "corresponding" dimensions (Melara \& O'Brien, 1987). In previous Garner-paradigm studies of rectangles, either data from the positive and negative correlated conditions have not been reported separately (Dykes, 1979), or such a difference has not been found (Dykes \& Cooper, 1978; Felfoldy, 1974). According to our models, the correspondence pattern arises directly from mean integrality, and we have no ready explanation for its nonoccurrence in earlier studies.

What about size and shape? In Krantz and Tversky's (1975) MDS experiment, these dimensions, as well as height and width, were found to interact. This interaction can in principle be assessed from our data by noting whether the perceptual dimensions of size and shape are orthogonal. However, our stimulus sets are not quite appropriate for inferring these dimensions: Stimuli $B$ and $C$ were not always chosen to have exactly the same size, or Stimuli $A$ and $D$ exactly the same shape (height/width ratio). If one examines the representations in Figures 3 and 6, it appears plausible that lines connecting points of equal size and shape in the perceptual space would be orthogonal, but a definitive answer requires the use of a stimulus set in which size and shape vary independently.

\section{Is Perception of Rectangles "Holistic"?}

The Garner paradigm and related techniques have long provided fodder for a holistic-versus-piecemeal dispute. Our data address two distinct aspects of this contrast: the distinctions between stimulus wholes and the dimensions on which they vary, and between stimulus wholes and their perceptual subsets.

Stimulus wholes versus dimensions. First, consider the present data in light of Lockhead's (1972) blob model, a popular instantiation of the holistic idea. According to the blob model, integral stimuli are first perceived in a unitary way (as blobs). Tasks that permit a judgment based on this unitary processing (i.e., baseline and correlated) are performed at a level determined by the discriminability of the stimuli in a perceptual space. Tasks that require judgments about individual dimensions (i.e., selective and divided attention) require more time. The model accounts for the usual integral syndrome, but allows for modifications resulting from different arrangements of the stimuli.

In most respects, our perceptual-space approach is quite consistent with the blob model and can indeed be viewed as a quantitative realization of it. Our baseline and correlated data are well described by a spatial model, in agreement with Lockhead's stress on the importance of the structure of the discriminability space. A difference in interpretation arises in considering the selective and divided attention tasks. According to Lockhead (1979), "the holistic model indeed does not account for the data in orthogonal [selective] tasks. This is because these are not identification tasks" (p. 750). Classification tasks are said to require an additional kind of processing; one possibility is that "the observer might erect a criterion in representational space" (p 751). The decline in performance might then be due to criterion variability, which is known to increase with stimulus set size.

In our analysis, no additional assumptions (such as a variance or criterion variability that increases with range) are needed to account qualitatively for filtering loss, or for the even greater loss that typically occurs in the divided task. Rather, optimal performance in these tasks is necessarily less than in the corresponding baseline tasks whenever mean integrality holds (Macmillan \& Kingston, 1995; Maddox, 1992). The important quantitative question is how the magnitudes of the observed and predicted losses compare. In selective attention, averaging across experiments and conditions, the predicted loss in percent correct was 2.2 points, the observed loss 3.1 ; the model predicts $71 \%$ of the observed decline. In divided attention, the predicted loss was 11.0 points and the observed loss 13.7 ; the model predicts $80 \%$ of the decline. In view of these results, one might ask whether the selective and divided "attention" tasks are well named, or whether they are, in fact, not very attention demanding.

A useful distinction can be drawn between extrinsic and intrinsic attention (Graham, 1989). The terms refer to the observer. Attention is extrinsic if performance can be accounted for in terms of the inherent limitations of the task on making judgments about the stimulus set; it is intrinsic if performance can only be accounted for with reference to limitations in the observer. Extrinsic performance is predicted from the optimal model of performance, whereas intrinsic constraints force accuracy below the optimal level. In the present study, it appears that most of the difference between the baseline and attention conditions is extrinsic, but a smaller fraction is due to some processing limitation in the observer.

Stimulus wholes versus parts. A second type of alternative to holistic perception is that distinct subsets of the stimuli--features, or components-are processed independently. The comparison between line pairs and rectangles in the present experiments allows us to test a 
specific, quantitative version of this hypothesis. In the Discussion to Experiment 2, we showed that the data were consistent with the idea that opposite line pairs combine independently in the perception of rectangle shape, but that the second pair added little to the perception of rectangle size. Thus size is perceived "holistically," in the sense that the stimulus is not decomposed perceptually, but shape is perceived "componentially." If this interpretation is correct, the answer to the partwhole question depends on the task to be performed, not just on the stimuli.

\section{Comments on the Perceptual-Space Approach}

We will close with two comments about the generality of the perceptual-space approach used in this paper, one concerning our dependent variable (accuracy), the other concerning our independent variables (the size, shape, height, and width of rectangles and line pairs).

The reliance of our approach on accuracy, rather than response time, is both a strength and a limitation. It is a strength because it provides quantitative measures of performance that can easily be compared with the models, and a weakness in that the ability of the model to address Garner paradigm results in the literature (most of which rely on response time) is limited. Ashby and Maddox (1994) have presented a response-time-based account of mean integrality, according to which response time and accuracy can be expected to covary negatively. Such covariation is commonly found in the literature, so it is plausible to interpret response time data collected within the Garner paradigm through the lens of our models. ${ }^{3}$ Response time data have often been used to support processing models, for which they are the natural grist, but we believe that such models are most convincing when built on a model of the perceptual representation. For example, there is no need for a process model to account for the difficulty of the divided attention task, because a decision theory analysis shows that even optimal performance in this task must be poorer than in the corresponding baseline conditions.

The parallelogram model of Garner data may be quite general. For example, we have applied it successfully to the perception of several dimensions of vowels. Kingston and Macmillan (1995) presented listeners with synthetic syllables in which the vowels varied in quality (between $/ \mathrm{o} /$ and $/ \mathrm{u} /$ ) and in nasalization. The inferred representation was mean-integral, and the value of $\theta$ was between $0^{\circ}$ and $44^{\circ}$ in different consonantal contexts. The RMSE for fits of the model was excellent, averaging 0.7 percentage points across five conditions. Kingston, Macmillan, Dickey, Thorburn, and Bartels (1997) studied vowels varying in quality (again in the /o/-/u/ region) over a wide range of voice tenseness/laxness. Two $4 \times 4$ stimulus arrays were used; each permitted nine tests of the model. Mean-integral representations were again found, with an average $R M S E$ of 2.0 percentage points for one set and 1.1 points for the other.
If the parallelogram model turns out to be generally applicable, it holds out the promise of a theoretical taxonomy of Garner paradigm results, based on parallelogram angle and perhaps other parameters. Such a taxonomy could provide a different perspective on perceptual interaction than the more familiar classification of dimensions as integral, separable, configural, corresponding, and so forth. At most, this approach has the potential to uncover the theoretical relations toward which the Garner tasks do, in fact, converge.

\section{REFERENCES}

Ashby, F. G., \& GotT, R. E. (1988). Decision rules in the perception and categorization of multidimensional stimuli. Journal of Experimental Psychology: Learning, Memory, \& Cognition, 14, 33-53.

Ashby, F, G., \& LEE, W. W. (1991). Predicting similarity and categorization from identification. Journal of Experimental Psychology: General, 120, 150-172.

Ashby, F. G., \& MADDOx, W. T. (1991). A response time theory of perceptual independence. In J. P. Doignon \& J. C. Falmagne (Eds.), Mathematical psychology: Current developments (pp. 389-413). New York: Springer-Verlag.

AshbY, F. G., \& MADDox, W. T. (1994). A response time theory of separability and integrality in speeded classification. Journal of Mathematical Psychology, 38, 423-466.

Ashry, F. G., \& Perrin, N. A. (1988). Toward a unified theory of similarity and recognition. Psychological Review, 95, 124-150.

ASHBY, F. G., \& TownSEND, J. T. (1986). Varieties of perceptual independence. Psychological Review, 93, 154-179.

BORG, I., \& LEUTNER, D. (1983). Dimensional models for the perception of rectangles. Perception \& Psychophysics, 34, 257-267.

DorfMAN, D. D., \& ALF, E., JR. (1969). Maximum likelihood estimation of parameters of signal detection theory and determination of confidence intervals - - Rating method data. Journal of Mathematical Psychology, 6, 487-496.

DuNN, J. C. (1983). Spatial metrics of integral and separable dimensions. Journal of Experimental Psychology: Human Perception \& Performance, 9, 242-257.

DYKES, J. R., JR. (1979). A demonstration of selection of analyzers for integral dimensions. Journal of Experimental Psychology: Human Perception \& Performance, 5, 734-745.

DYKes, J. R., JR., \& CoOPER, R. G., JR. (1978). An investigation of the perceptual basis of redundancy gain and orthogonal interference for integral dimensions. Perception \& Psychophysics, 23, 36-42.

FeLFOLDY, G. L. (1974). Repetition effects in choice reaction time to multidimensional stimuli. Perception \& Psychophysics, 15, 453-459.

GARNER, W. R. (1974). The processing of information and structure. Potomac, MD: Erlbaum.

GARNER, W. R., \& FelFOLDY, G. L. (1970). Integrality of stimulus dimensions in various types of information processing. Cognitive Psychology, 1, 225-241.

Gourevitch, V., \& Galanter, E. (1967). A significance test for oneparameter isosensitivity functions. Psychometrika, 32, 25-33.

Graham, N. V. (1989). Visual pattern analyzers. New York: Oxford University Press.

KADLEC, H. (1995). Multidimensional signal detection analyses (MSDA) for testing separability and independence: A Pascal program. Behavior Research Methods, Instruments, \& Computers, 27, 442-458.

KADLEC, H., \& ToWNSEND, J. T. (1992). Signal detection analyses of dimensional interactions. In F. G. Ashby (Ed.), Multidimensional models of perception and cognition (pp. 181-227). Hillsdale, NJ: Erlbaum.

KinGSTON, J., \& MACMILLAN, N. A. (1995). Integrality of nasalization and $F 1$ in vowels in isolation and before oral and nasal consonants: A detection-theoretic application of the Garner paradigm. Journal of the Acoustical Society of America, 97, 1261-1285. 
Kingston, J., Macmillan, N. A., Dickey, L. W., Thorburn, R., \& BARTEL,S, C. (1997). Integrality in the perception of tongue root position and voice quality in vowels. Journal of the Acoustical Society of America, 101, 1696-1709.

KrantZ, D. J., \& TVERSKy, A. (1975). Similarity of rectangles: An analysis of subjective dimensions. Journal of Mathematical Psychology, 12, 4-34.

KrUMHANSL, C. L. (1978). Concerning the applicability of geometric models to similarity data: The interrelationship between similarity and spatial density. Psychological Review, 85, 445-463.

LaZARTE, A. A., \& SchönemanN, P. H. (1991). Saliency metric for subadditive dissimilarity judgments of rectangles. Perception $\&$ Psychophysics, 49, 142-158.

LOCKHEAD, G. R. (1970). Identification and the form of multidimensional discrimination space. Journal of Experimental Psychology, 85, $1-10$.

LOCKhEad, G. R. (1972). Processing dimensional stimuli: A note. Psychological Review, 79, 410-419.

LOCKHEAD, G. R. (1979). Holistic versus analytic process models: A reply. Journal of Experimental Psychology: Human Perception \& Performance, 5, 746-755.

LockHEAd, G. R., \& KıNG, M. C. (1977). Classifying integral stimuli. Journal of Experimental Psychology: Human Perception \& Performance, 3, 436-443.

Macmillan, N. A., \& Creelman, C. D. (1991). Detection theory: A user's guide. New York: Cambridge University Press.

MaCMillan, N. A., \& Kingston, J. (1995). Integrality, correspondence, and configurality represent different degrees of perceptual interaction, not different types. In C.-A. Possamai (Ed.), Fechner Day 95 (pp. 243-248). Cassis, France: International Society for Psychophysics.

MadDox, W. T. (1992). Perceptual and decisional separability. In F. G, Ashby (Ed.), Multidimensional models of perception and cognition (pp. 147-180). Hillsdale, NJ: Erlbaum.

MadDox, W. T., \& Ashby, F. G. (1996). Perceptual separability, decisional separability, and the identification-speeded classification relationship. Journal of Experimental Psychology: Human Perception \& Performance, 22, 795-817.

Marascuilo, L. A. (1970). Extensions of the significance test for oneparameter signal detection hypotheses. Psychometrika, 35, 237-243.

Melara, R. D., \& O'Brien, T. P. (1987). Interaction between synesthetically corresponding dimensions. Journal of Experimental Psychology: General, 116, 323-336.

MONAHAN, J. S., \& LOCKHEAD, G. R. (1977). Identification of integral stimuli. Journal of Experimental Psychology: General, 106, 94-110.

POSNER, M. I. (1964). Information reduction in the analysis of sequential tasks. Psychological Review, 71, 491-504.

SCHÖNEMANN, P. H. (1990). Psychophysical maps for rectangles. In H.-G. Geissler (Ed.), Psychophysical explorations of mental structures (pp. 149-164). Toronto: Hogrefe \& Huber.

Schönemann, P. H., DorCEY, T., \& KienapPle, K. (1985). Subadditive concatenation in dissimilarity judgments. Perception \& Psychophysics, 38, 1-17.

Schönemann, P. H., \& Lazarte, A. (1987). Psychophysical maps for subadditive dissimilarity ratings. Perception \& Psychophysics, 42, 342-354.

ShEPARD, R. N. (1964). Attention and the metric structure of the stimulus space. Journal of Mathematical Psychology, 1, 54-87.

Swets, J. A., \& Picketr, R. M. (1982). Evaluation of diagnostic sys- tems: Methods from signal detection theory. New York: Academic Press.

Weintraub, D. J. (1971). Rectangle discriminability: Perceptual relativity and the law of Prägnanz. Journal of Experimental Psychology, 88, 1-11.

WENDER, K. (1971). A test of independence of dimensions in multidimensional scaling. Perception \& Psychophysics, 10, 30-32.

WIENER-EHRLICH, W. K. (1978). Dimensional and metric structures in multidimensional stimuli. Perception \& Psychophysics, 24, 399-414.

\section{NOTES}

1. The terms integral and separable have long been used to refer to pairs of dimensions that are interacting versus those that are independent. In the context of the Garner paradigm, these terms refer operationally to whether dimension pairs exhibit a filtering loss and redundancy gain. When interaction or independence is assessed theoretically from an inferred perceptual representation, we use the terms introduced by generalized recognition theory: mean-integral (Maddox, 1992) and perceptually separable (Ashby \& Townsend, 1986). The former term could be more precisely rendered as perceptually mean-integral, but this seems unnecessarily cumbersome.

2. The multidimensional models used in this paper assume that the underlying bivariate distributions are (1) Gaussian, (2) of equal variance (i.e., when the four distributions are projected onto the horizontal axis-or the vertical axis-the variances of all distributions are the same), and (3) uncorrelated. This last assumption is termed perceptual independence (Ashby \& Maddox, 1991; Ashby \& Townsend, 1986). Nested models (Ashby \& Lee, 1991) are sometimes used to evaluate assumptions of this type, but there are too few degrees of freedom in the Garner paradigm studies to do this. What evidence do we have that these assumptions are correct?

Assumptions 1 and 2 predict that the ROC curves in the two-stimulus conditions should be straight lines with unit slope, and to a first approximation they are. Assumption 3 could be violated in two ways: (a) the correlations could be nonzero and equal, and (b) at least some of the correlations could be nonzero, and not all of them equal. Case (a) is not a problem, because a representation in which all correlations (and variances) are equal is equivalent to one in which all correlations are zero but the axes are nonorthogonal (see Ashby \& Townsend, 1986, Figure 5). Nonorthogonality of the axes appears to be the same as mean integrality, in our application.

Case (b) would be a surprising outcome: After all, the two elements (height and width) that compose a stimulus are very close to the same for all four possibilities, so one would expect any correlation to be similar for all. But if it is true, our analysis is misleading, for unequal amounts of correlation can lead to violations of the distance axioms (Ashby \& Perrin, 1988). In defense of our approach, such violations should lead to poor fits of the parallelogram model to the data, and our fits were very good. Small differences in correlation may have occurred, but seem unlikely to have substantially affected our conclusions.

3. This argument applies Ashby and Maddox's (1994) "RT-distance hypothesis" qualitatively. If assumptions about the form of the function relating $\mathrm{RT}$ and distance are made, the hypothesis can be used to make quantitative predictions (Maddox \& Ashby, 1996).

(Manuscript received April 12, 1996; revision accepted for publication February 10, 1997.) 